\title{
Tunable unipolar synchronized electric charge extraction strategy for piezoelectric energy harvesting
}

Journal of Intelligent Material Systems and Structures

$1-10$

(c) The Author(s) 2019

Article reuse guidelines:

sagepub.com/journals-permissions DOI: 10.1 I 77// 045389X19844329

journals.sagepub.com/home/jim @SAGE

\author{
Alexis Brenes' ${ }^{(\mathbb{D},}$, Elie Lefeuvre', Seonho Seok' and Chan-Sei Yoo ${ }^{2}$
}

\begin{abstract}
This article focuses on an intelligent control strategy to improve the performances of shunt-rectifier architectures for vibration energy harvesting. It demonstrates how proper tuning can improve the frequency bandwidth and maximum power of unipolar synchronized electric charge extraction architectures. For resonators with strong enough coupling $\left(k^{2} Q>\pi / 2\right)$, tuning the duration of charge extraction with the oscillation frequency improves the power harvesting performances. The main differences with other similar solutions such as unipolar synchronized electric charge extraction without tuning strategy or tunable synchronized electric charge extraction are illustrated. In particular, we show how the choice of the shunt rectifier significantly affects the power response of the generator due to electromechanical coupling phenomenon. The analytical study is experimentally validated on a cantilever-based piezoelectric generator.
\end{abstract}

\section{Keywords}

Energy harvesting, piezoelectric transducers, unipolar synchronized electric charge extraction

\section{Introduction}

In the field of vibration energy harvesting, smart structures based on piezoelectric material play a major role because of their high power density compared to other technologies. Resonant systems are known to be a good candidate for frequency-specific applications because they provide optimal performance when properly designed for the working frequency. However, unless specific control strategies are developed, their performance remains limited by their intrinsically narrow bandwidth, essentially determined by the mechanical characteristics of the generator.

Among the strategies to optimize the harvested power, synchronized switching methods are good candidates due to their performances and limited complexity (Boussaid, 2015; Lien et al., 2010; Sanchez et al., 2016). Self-powered systems based on synchronized switching even already exist (Lallart, 2017). Yet, for these strategies to be fully efficient, a control loop is required. For example, parallel and series synchronized-switch harvesting on inductor (p-SSHI and s-SSHI) require maximum power point tracking (MPPT) adapted to the frequency of the mechanical oscillation (Lien et al., 2010). This tracking can, for example, be obtained by adjustment of the duty-cycle or switching frequency of a DC-DC converter (Shu and Lien, 2006). For this reason, the optimal design of
SSHI architectures requires two different control stages to be fully efficient: one dedicated to the synchronized switching and the other controlling switches at the DCDC converter stage ensuring impedance matching.

Contrary to the aforementioned SSHI strategies, another category exists where the control of harvested power only requires one single synchronous control stage (Guyomar and Lallart, 2011; Lefeuvre et al., 2005). These strategies are usually called synchronized electric charge extraction (SECE), where the control of synchronized switching itself maximizes the power flow from the mechanical side to the electronics. Optimized variants of the original SECE (called 'classical SECE') include tunable SECE (Lefeuvre et al., 2017), phase-shift SECE (PS-SECE) (Lefeuvre et al., 2017), frequencytuning SECE (FTSECE) (Badel and Lefeuvre, 2016), multi-shot SECE (Gasnier et al., 2014) or optimized SECE (OSECE) (Wu et al, 2014). Among these, tunable SECE (Lefeuvre et al., 2017a; Richter et al., 2014) has

\footnotetext{
'Centre for Nanoscience and Nanotechnology, University of Paris Sud, CNRS, Université Paris-Saclay, Orsay, France

${ }^{2}$ Electronic Convergence Materials \& Device Research Center, Korea Electronics Technology Institute, Republic of Korea
}

\section{Corresponding author:}

Alexis Brenes, Centre for Nanoscience and Nanotechnology, Univ. Paris Sud - CNRS, Université Paris-Saclay, Orsay 91405, France.

Email: alexis.brenes@u-psud.fr 


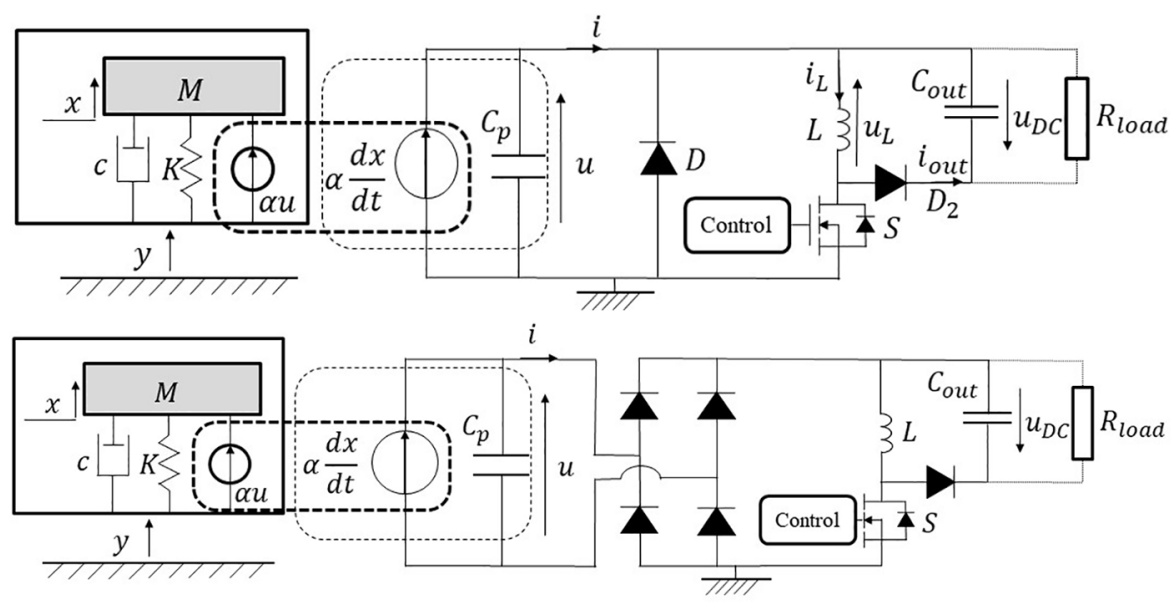

Figure I. SDOF linear electromechanical model of the inertial piezoelectric energy harvester and electronic circuit of (tunable) USECE (top) and classical (or tunable) SECE (bottom).

demonstrated strong improvements to the original SECE in terms of harvested power.

However, in standard SECE architectures, the electrical power efficiency is limited at low vibration levels by the threshold voltage of the diodes used in the fullbridge rectifier (FB rectifier) stage. To confront this issue, a recent simple solution called 'unipolar SECE' (USECE) takes advantage of a single-diode shunt rectifier instead of a FB rectifier (Brenes et al., 2018). In this article, this originally published USECE (Brenes et al., 2018) will be referred to as 'classical USECE'. It has been demonstrated that the shunt-diode rectifier of the originally published USECE strongly impacts the overall electromechanical behaviour of the system, not only through its power efficiency at low levels but also in terms of impedance matching. Although seemingly simple, such a modification impacts the behaviour of the system due to the bidirectional piezoelectric coupling. Except in very specific situations (e.g. simultaneously very weak-coupling and high-voltage amplitude), classical USECE has been proven capable of harvesting more power than classical SECE due to the combination of a larger input power and an enhanced power efficiency. However, classical USECE can only reach the theoretical optimum in terms of harvested power for very specific resonators (in terms of figure of merit $k^{2} Q(\mathrm{FOM})$ ), and its frequency bandwidth remains limited (Brenes et al., 2018).

In this article, we adapt the tuning strategy of tunable SECE to classical USECE, by tuning the duration of charge extraction depending on the working frequency. The resulting scheme is called 'tunable USECE'. We show the results of this modification in terms of maximum power and system bandwidth. We also demonstrate experimentally how tunable USECE improves the harvesting performances of an USECE architecture.
This article is organized as follows. First, we recall the electronic architecture of classical USECE and explain the main differences introduced by the tuning approach. We show how the control strategy impacts the system through the electromechanical coupling. Then, the performance of tunable USECE is experimentally demonstrated on a piezoelectric cantilever resonator.

\section{Study of the tunable USECE}

\section{I. Model of inertial piezoelectric device}

The architecture of tunable USECE is identical to the one previously published for classical USECE (Brenes et al., 2018) except that the control scheme (presented in this section) is different. The circuit of classical (and tunable) SECE is also reminded in Figure 1, for comparison.

Before going further, one must remember that the optimization of energy harvesters requires to consider two complementary aspects. First, to maximize the harvested power, one must optimize the electrical power efficiency (i.e. minimize the electrical losses). This was, for instance, the purpose of the single-diode shunt architecture of USECE compared to the full-bridge architecture of classical SECE (Brenes et al., 2018). Second, one must try to optimize the input power, that is, the power extracted from the piezoelectric material at a given acceleration level (independently of the electrical power efficiency). This can be done with a specifically designed control scheme. Optimizing both the power efficiency and the input power is the only way to guarantee that the energy harvester is fully optimized. In this article, we will focus on this second aspect because the problem of electrical power efficiency of the shunt-diode architecture combined with SECE has already been addressed. We focus on the next step: maximizing, based on the architecture of USECE, the 
Table I. Variables and normalizations.

\begin{tabular}{|c|c|c|}
\hline Variable & Quantity (unit) & $\begin{array}{l}\text { Normalized } \\
\text { variable }\end{array}$ \\
\hline$\omega$ & $\begin{array}{l}\text { Vibration angular } \\
\text { frequency }\left(\mathrm{rad} . \mathrm{s}^{-1}\right)\end{array}$ & $\Omega=\frac{\omega}{\omega_{0}}$ \\
\hline$y$ & Displacement of the base $(\mathrm{m})$ & $Y=\frac{y \omega_{0}^{2}}{\gamma_{m}}$ \\
\hline$x$ & $\begin{array}{l}\text { Displacement of } \\
\text { the resonator }(\mathrm{m})\end{array}$ & $X=\frac{x \omega_{0}^{2}}{\gamma_{m}}$ \\
\hline$p$ & Piezoelectric output power (W) & $P=\frac{p}{p \lim } Q=\frac{8 p \omega_{0}}{M \gamma_{m}^{2}}$ \\
\hline$i$ & Piezoelectric current (A) & $I=\frac{i \alpha}{C_{p} M \omega_{0} \gamma_{m}}$ \\
\hline$u$ & Piezoelectric voltage $(\mathrm{V})$ & $U=\frac{u \alpha}{M \gamma_{m}}$ \\
\hline
\end{tabular}

power extracted from the piezoelectric generator with an optimal control scheme.

To understand how a tunable USECE circuit behaves, one must estimate the power extracted from a piezoelectric generator by the architecture. The study is based on the model of a single-degree-of-freedom resonant mass, depicted in Figure 1, electromechanically coupled to the USECE architecture. In the considered model, a mass $M$ is suspended by a spring of stiffness $K$. The natural angular frequency is thus $\omega_{0}=\sqrt{K / M}$. The mechanical losses are modelled by the damper $c$. Vibrations of the environment give rise to a mechanical displacement $y$ of the resonator base which actuates the motion of the inertial mass with respect to the base. The relative displacement of mass $M$ with respect to the base is written as $x$. We assume that the piezoelectric resonator is subject to a harmonic acceleration $d^{2} y / d t^{2}=\gamma(t)=\gamma_{m} \cos (\omega t+\phi)$.

The electromechanical coupling is accounted for by the factor $\alpha\left(\mathrm{N} . \mathrm{V}^{-1}\right)$. Because of this coupling, any modification on the electrical side (respectively, mechanical) affects the behaviour on the mechanical side (respectively, electrical). On the electrical side, the capacitance of the piezoelectric element is written as $C_{p}$. The diode $D$ placed across the resonator makes the piezoelectric voltage $u$ unipolar, similarly to what is sometimes called a class-E zero-voltage-switching rectifier (Kazimierczuk, 1990). With the aforementioned notations, the equations governing the system behaviour are (equation (1))

$$
\left\{\begin{array}{c}
M \frac{d^{2} x}{d t^{2}}+c \frac{d x}{d t}+K x+\alpha u=-M \gamma_{m} \cos (\omega t+\phi) \\
i=\alpha \frac{d x}{d t}-C_{p} \frac{d u}{d t}
\end{array}\right.
$$

Many parameters appear in equation (1), but only a limited number is required to describe and understand the behaviour of the system (Arroyo et al., 2012). The relevant parameters can be highlighted after the normalization given in Table 1, which allows us to rewrite equation (1) as equation (2). One of the relevant parameters, $M \omega_{0} / c$, is the mechanical quality factor of the resonator which appears in equation (2)

$$
\left\{\begin{array}{l}
\frac{d^{2} x}{d t^{2}}+\frac{\omega_{0}}{Q} \frac{d x}{d t}+\omega_{0}^{2} x+\frac{\alpha}{M} u=-\gamma_{m} \cos (\omega t+\phi) \\
\frac{i \alpha}{C_{p} M \gamma_{m}}=\frac{\alpha^{2}}{C_{p} M \gamma_{m}} \frac{d x}{d t}-\frac{\alpha}{M \gamma_{m}} \frac{d u}{d t}
\end{array}\right.
$$

Finally, equation (2) becomes equation (3), where $k_{m}^{2}=\alpha^{2} / K C_{p}$ is the modified electromechanical coupling coefficient and the angle $\theta$ is defined by $\theta=\omega_{0} t$

$$
\left\{\begin{array}{c}
\frac{d^{2} X}{d \theta^{2}}+\frac{1}{Q} \frac{d X}{d \theta}+X+U=-\cos (\Omega \theta+\phi) \\
I=k_{m}^{2} \frac{d X}{d \theta}-\frac{d U}{d \theta}
\end{array}\right.
$$

Now that the model and the electronic architecture have been presented, the next section introduces the control scheme of tunable USECE.

\subsection{Control scheme for tunable USECE}

In the following discussion, we will assume that the piezoelectric voltage amplitude is large enough to neglect the impact of the threshold voltage $u_{D}$ of diode $D$ on the power delivered by the generator into the circuit. In most applications, this assumption remains valid for USECE architectures, and the impact of $u_{D}$ on the power delivered by the generator into the circuit remains quite small (see, for example, Brenes et al., 2018). If needed, the model presented in this article can be extended to take into account $u_{D}$. The resulting derivations are quite straightforward but yield more complex expressions.

As long as the switch $S$ is open and the voltage is positive, the piezoelectric generator remains in open circuit. In tunable USECE, as in classical USECE, the switch $S$ is closed once a period, when the piezoelectric voltage $u$ reaches its peak value $u_{\text {peak }}$. The time interval during which the switch $S$ is closed is called the 'charge extraction phase'. Then, the switch $S$ is re-opened and the generator remains in open circuit until $u$ cancels out. From that moment to the next period, the resonator remains short-circuited by the shunt diode $D$.

The charge extraction phase consists in a quasiinstantaneous LC-oscillation between the piezoelectric capacitance and the inductor $L$ (see Figure 1), which is chosen small enough to neglect the LC-time constant compared to the oscillation period $T$ (i.e. $2 \pi \sqrt{L C_{p}} \ll T$ ). In tunable USECE, contrary to classical USECE, the duration of that charge extraction phase is tuned. This electrical degree-of-freedom introduced by the tuning strategy is called the 'tuning parameter' and is written as $\beta$. It is defined in such a way that, at the end of the charge extraction phase, the piezoelectric voltage is $u=\beta u_{\text {peak }}$ (with $\beta \in[0,1]$ ). Typical waveforms of the piezoelectric voltage $u$ and the displacement $x$ are depicted in Figure 2. As we will see, this tuning parameter $\beta$ can be optimized to maximize the harvested power.

Then, the piezoelectric voltage remains null until the following period. As in the classical USECE circuit, the 

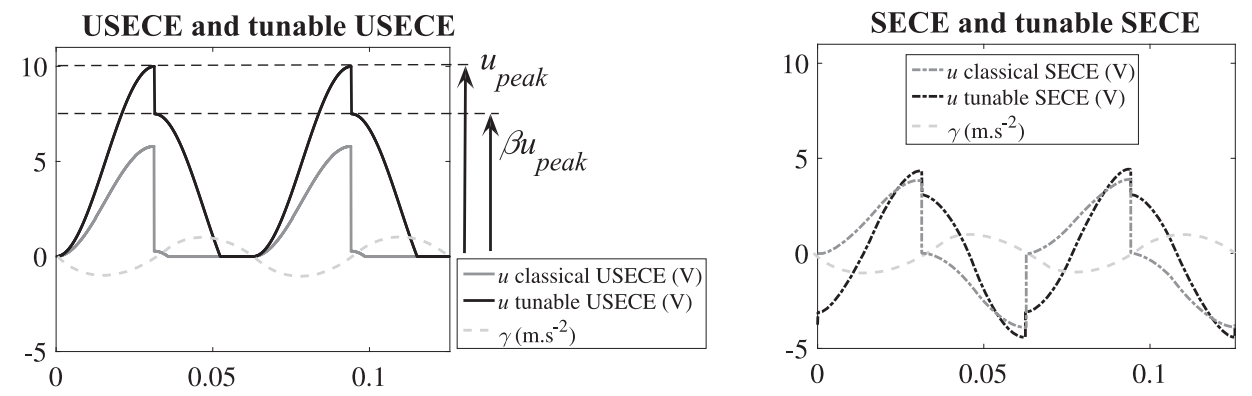

Figure 2. Typical waveforms close to resonance. Piezoelectric voltage $u$ (dark) and acceleration $\gamma$ (grey) for $\beta=0.7$. Left: Comparison between tunable USECE (solid dark line) and classical USECE (solid grey line). Right: Comparison with the waveforms of classical SECE and tunable SECE.

shunt rectifier is OFF during the charge extraction phase (where the peaks of current occur), contrary to SECE. This ensures a good power efficiency even at low voltages (Brenes et al., 2018).

Before writing down the mathematical formulation, one can already discuss qualitatively the main idea of the tuning strategy. For $\beta=0$ (case of classical USECE), all the electrical energy stored in the piezoelectric capacitor is extracted. Thus, this situation seems optimal from an electrical point of view. However, $\beta=0$ also corresponds to a high equivalent electrical damping, which thus reduces the amplitude of the mechanical motion due to the reverse piezoelectric effect.

Compared to this situation where $\beta=0$, an increase of $\beta$ (i.e. shortening the charge extraction phase) diminishes the fraction of electrical energy periodically extracted, which is seemingly non-optimal from an electrical perspective. However, in the same time, it increases the available power by increasing the oscillation amplitude through a reduction of the equivalent damping. As in tunable SECE (Lefeuvre et al., 2017), there is an optimal value of $\beta$ in tunable USECE to strike a balance between increasing the overall energy available and increasing the fraction of energy extracted at each period. This trade-off between harvested energy and additional damping is not new in the field of energy harvesting and has already been discussed in the case of other harvesting strategies (Lallart, 2017; Lesieutre et al., 2004; Yoshimizu et al., 2017).

One may notice in Figure 2 that, close to resonance, tunable USECE reaches the highest piezoelectric voltages (compared to classical USECE, classical SECE and tunable SECE). This is due to two main reasons. First, compared to classical USECE, the amplitude of the mechanical motion reached by tunable USECE is larger at resonance because, for a proper tuning of $\beta$, the damping introduced by a shorter charge extraction becomes smaller. One way to understand why the piezoelectric voltage is higher in tunable schemes is to realize that the active switching actually retroacts constructively on the mechanical oscillation. The control scheme allows adjusting the phase-shift between the piezoelectric current and the piezoelectric voltage while minimizing the real part of the input impedance of the circuit. Because of this, more mechanical power coming from the base movement is transferred into mechanical power at the seismic mass level. Thus, for an identical acceleration of the base $\gamma(t)$, the amplitude of the motion of the seismic mass $M$ is higher than in the case of non-tunable schemes. Such a larger mechanical motion leads to higher piezoelectric voltages. One can then afford to extract a smaller portion of the total available energy and still get more power at the input of the electronic circuit.

Second, in tunable USECE, there is a positive DC component, which leads to a higher peak voltage than in classical or tunable SECE (with the FB rectifier). The combination of these two phenomena (increased oscillation amplitude due to limited electrical damping and non-cancellation of the DC component) explains why tunable USECE reaches the highest piezoelectric voltages close to resonance.

\subsection{Analysis of tunable USECE}

For the analysis, we write the motion $X=-X_{m} \cos \Omega \theta$, the higher harmonics being filtered out due to the high mechanical quality factor of the resonator (Liao and Sodano, 2008). To deduce the harvested power, one must find out the relationship between the (normalized) piezoelectric voltage $U$ and the (normalized) displacement $X$. Based on the waveforms of Figure 2 and the coupled system of equation (3), the voltage $U$ can be written (equation (4)) in the time domain

$$
\left\{\begin{array}{l}
U(\theta)=k_{m}^{2} X_{m}(1-\cos \Omega \theta) \\
\quad \text { for } 0<\theta<\frac{\pi}{\Omega} \\
U(\theta)=k_{m}^{2} X_{m}(-\cos \Omega \theta+2 \beta-1) \\
\text { for } \frac{\pi}{\Omega}<\theta<\frac{1}{\Omega}[2 \pi-\arccos (2 \beta-1)] \\
U(\theta)=0 \\
\text { for } \frac{1}{\Omega}[2 \pi-\arccos (2 \beta-1)]<\theta<\frac{2 \pi}{\Omega}
\end{array}\right.
$$

The peak piezoelectric voltage (normalized as in Table 1 ) is given by equation (5) and is achieved for $\theta=\pi / \Omega$ 


$$
U_{\text {peak }}=\max (U)=2 k_{m}^{2} X_{m}
$$

The average power $p$ extracted from the piezoelectric transducer over each energy conversion cycle is given by equation (6)

$$
p=\frac{\omega}{4 \pi} C_{p} u_{p e a k}^{2}\left(1-\beta^{2}\right)
$$

As in the previous work (Lefeuvre et al., 2017), we define the normalized extracted power by $P=p Q / p_{\text {lim }}$, where $p_{\text {lim }}$ is the maximum average power which can be harvested by the piezoelectric energy harvester (see equation (7)) (Halvorsen et al., 2013)

$$
p_{\text {lim }}=\frac{M \gamma_{m}^{2}}{8 \omega_{0}} Q
$$

This normalization leads to equation (8)

$$
P=\frac{2 \Omega}{\pi k_{m}^{2}} U_{\text {peak }}^{2}\left(1-\beta^{2}\right)
$$

Hence, the input power is written as equation (9)

$$
P=\frac{8 \Omega k_{m}^{2} X_{m}^{2}}{\pi}\left(1-\beta^{2}\right)
$$

(9) From equation (11), one obtains the normalized amplitude $X_{m}$ given by equation (12)

$$
X_{m}^{2}=\frac{1}{\left[1-\Omega^{2}+\frac{k_{m}^{2}}{2 \pi}[\pi-(1-2 \beta) \sqrt{2 \beta(2-2 \beta)}+\arccos (1-2 \beta)]\right]^{2}+\left[\frac{\Omega}{Q}+\frac{2 k_{m}^{2}}{\pi}\left[1-\beta^{2}\right]\right]^{2}}
$$

Finally, equation (13), derived from equations (9) and (12), gives the power delivered by the piezoelectric generator into the circuit

$$
P=P_{o p t}=\frac{8}{\pi} \frac{k_{m}^{2} \Omega\left(1-\beta^{2}\right)}{\left[1-\Omega^{2}+\frac{k_{m}^{2}}{2}\left[1-\frac{2}{\pi}(1-2 \beta) \sqrt{\beta(1-\beta)}+\frac{1}{\pi} \arccos (1-2 \beta)\right]\right]^{2}+\left(\frac{\Omega}{Q}+\frac{2 k_{m}^{2}}{\pi}\left(1-\beta^{2}\right)\right)^{2}}
$$

Equation (13) is significantly different from the expression (14) obtained for tunable SECE (Lefeuvre et al., 2017)

$$
P_{\text {tunable SECE }}=\frac{16}{\pi} \frac{1-\beta}{1+\beta} \frac{k_{m}^{2} \Omega}{\left(1-\Omega^{2}+k_{m}^{2}\right)^{2}+\left(\frac{\Omega}{Q}+k_{m}^{2} \cdot \frac{4}{\pi} \cdot \frac{1-\beta}{1+\beta}\right)^{2}}
$$

From the comparison between equations (13) and (14), one can see that tunable USECE harvests half the power of tunable SECE for very small coupling coefficients because the harvesting events of tunable USECE take place only once a period. A further comparison shows that tunable USECE tunes simultaneously the electrical stiffness and the electrical damping, which is not the case in tunable SECE where $\beta$ only tunes the electrical damping. This explains why, for stronger coupling, $\beta$ can be tuned to compensate for this loss by enlarging the mechanical motion. In terms of power, considering the relatively complex expression of $P$, finding the condition on $k_{m}^{2}$ and $Q$ ensuring that the power reaches the maximum achievable power $p_{\text {lim }}$ (i.e. $P=Q)$ is not straightforward. However, numerical tests have shown that the condition remains the same as for classical USECE, which is $k^{2} Q \geqslant \pi / 2$, where $k^{2}=k_{m}^{2} / 1+k_{m}^{2}$. The product $k^{2} Q$ is usually referred to as a piezoelectric FOM.

As long as $k^{2} Q \geqslant \pi / 2$, the system harvests the maximum available power at one specific frequency. This is illustrated in Figure 3, where we compare the power 

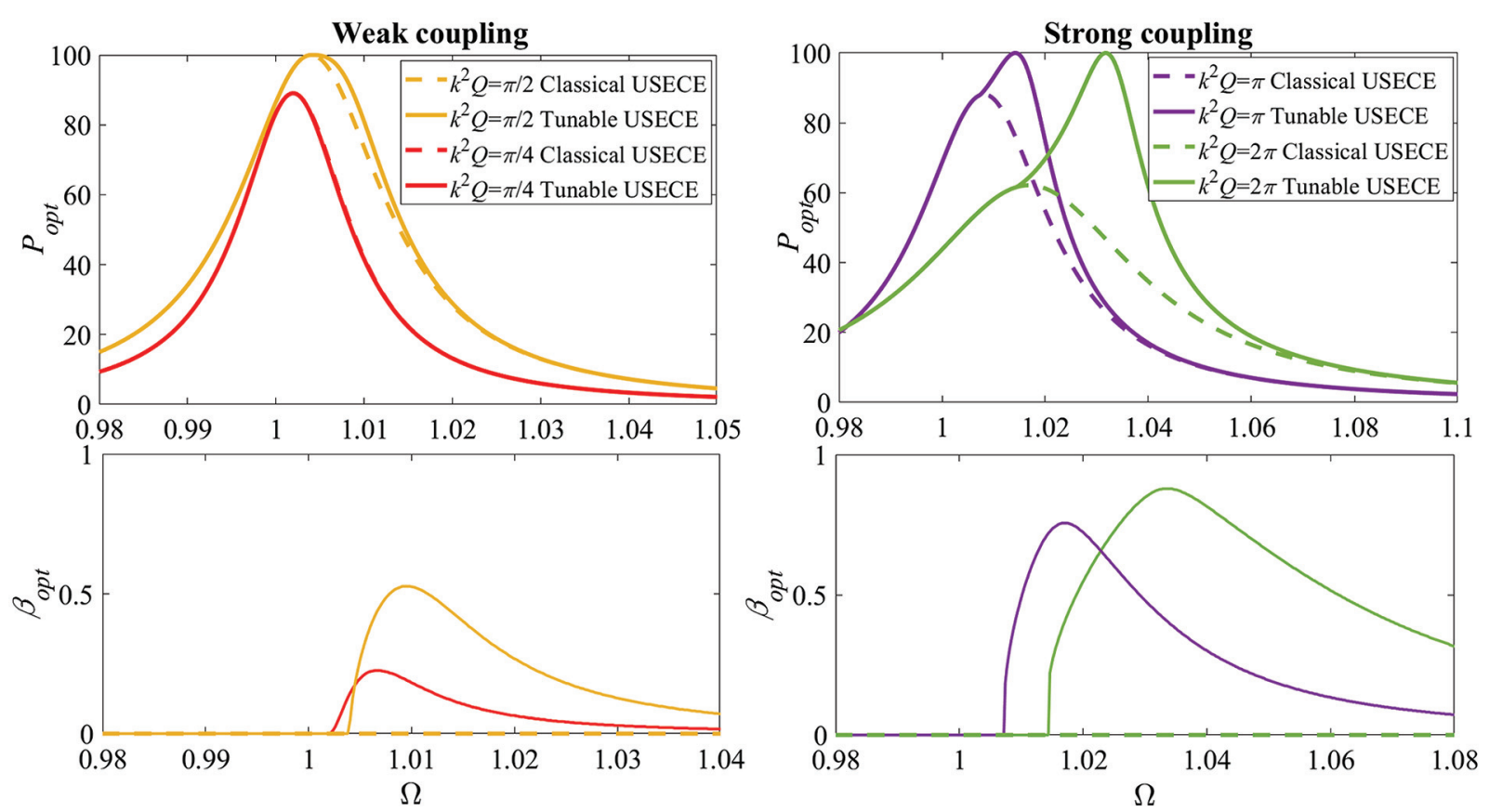

Figure 3. Comparison between tunable USECE and classical USECE with $Q=100$. Dashed lines indicate classical USECE $(\beta=0)$ (Brenes et al., 2018) and solid lines indicate tunable USECE.

versus frequency responses of tunable USECE with those of classical USECE, for several coupling levels. For weak coupling $\left(k^{2} Q<\pi / 2\right)$, almost no improvement can be achieved by tuning $\beta$. However, for larger coupling coefficients, such tuning reaches two important aims. First, it ensures that the maximum available power is achieved at one frequency. Second, it enlarges the bandwidth of the system. Interestingly, the maximum power is achieved for relatively large values of $\beta$, which means that the voltage drop during the charge extraction phase becomes quite small $(\beta=1$ would correspond to no extraction at all). The upper harmonics of the piezoelectric voltage are thus minimized in the same time, and the risk of actuating parasitic vibrations modes $(\mathrm{Li}, 2011)$ is diminished compared to USECE.

\subsection{Bandwidth enhancement brought by tunable USECE compared to tunable SECE}

Since the operation of tunable USECE looks close to tunable SECE, one may also wonder about the comparison between these two. Before going further in the developments, we want to emphasize that the difference between the two lies both in the input power (power delivered by the generator to the circuit) and in the electrical power efficiency. In this section, we address the impact of the tunable USECE scheme on the input power because the advantages of USECE compared to SECE in terms of power efficiency have already been demonstrated (Brenes et al., 2018).

For the comparison, we have plotted, in Figure 4, the input power of tunable USECE and tunable SECE, for weak and strong coupling. In the weakest coupling case, the input power obtained with tunable USECE is quite low compared to tunable SECE. This drawback has already been pointed out in our previous study of USECE (Brenes et al., 2018). On the contrary, in the strong-coupling case, tunable USECE exhibits much better performances than tunable SECE in terms of $-3 \mathrm{~dB}$ bandwidth. This advantage gets stronger as the coupling increases $\left(+40 \%\right.$ for $\left.k^{2} Q=2 \pi\right)$.

\subsection{Analysis of maximum power}

To extend our analysis, we report, in this section, the evolution of the maximum power achieved with four different harvesting strategies. The evolution of the maximum value $P_{\max }$ achieved by $P_{\text {opt }}$ is plotted in Figure 5 with respect to the FOM $k^{2} Q$. The comparison is provided between the proposed tunable USECE, classical SECE, tunable SECE and classical USECE. As already said, one can check that, apart from very weak-coupling cases, tunable USECE and tunable SECE both achieve the maximum available power which corresponds to $P_{\max }=Q$. This is one of the biggest advantages of such tuning methods, compared to classical SECE (or classical USECE).

\subsection{Effect of a constant tuning factor on the energy harvesting performances}

From an electronic perspective, the implementation of a tunable USECE interface may be complex and energy-consuming since it requires intelligent control of 

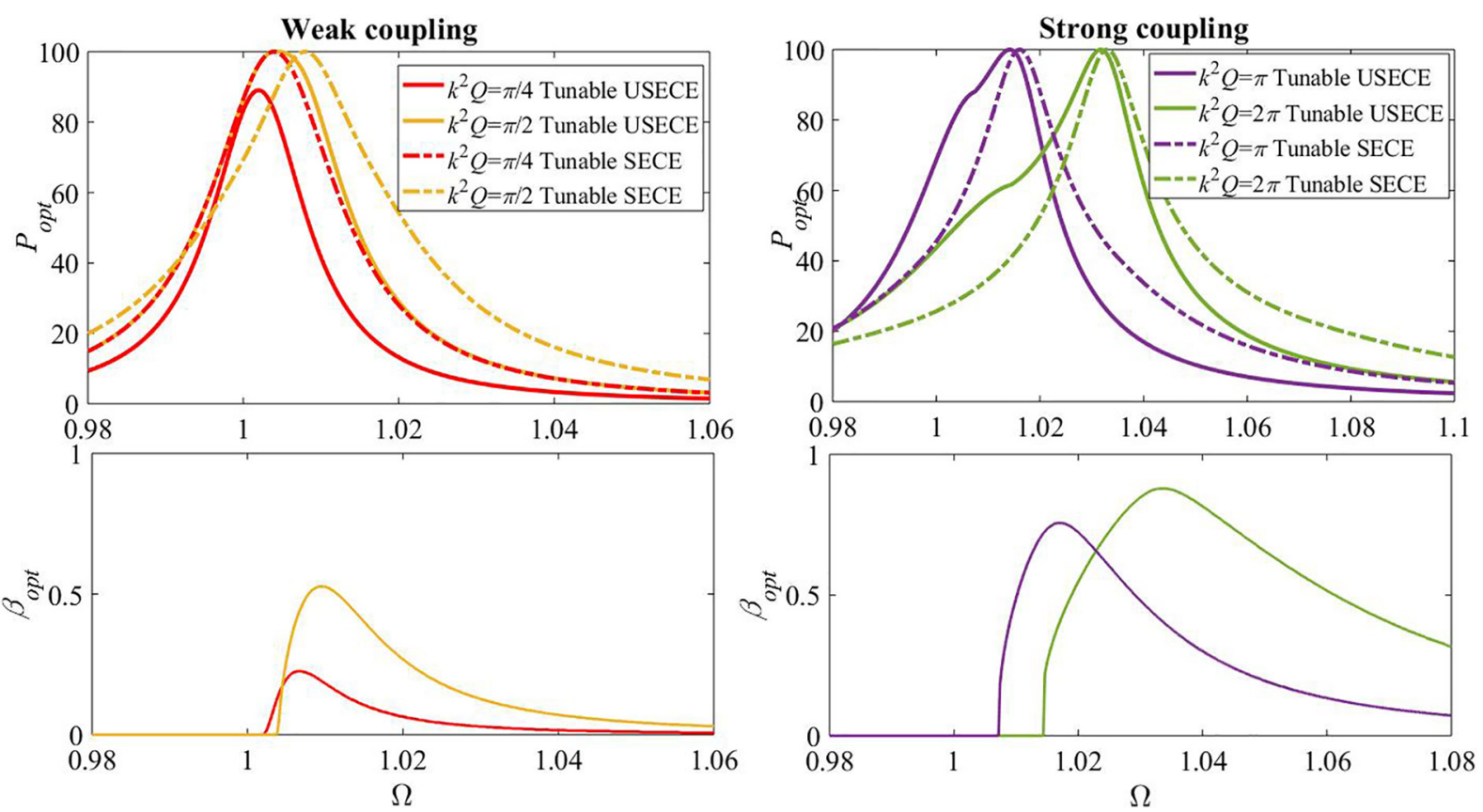

Figure 4. Comparison between tunable USECE and tunable SECE with $Q=100$. Dash-dotted lines indicate tunable SECE (Lefeuvre et al., 20I7) and solid lines indicate tunable USECE.

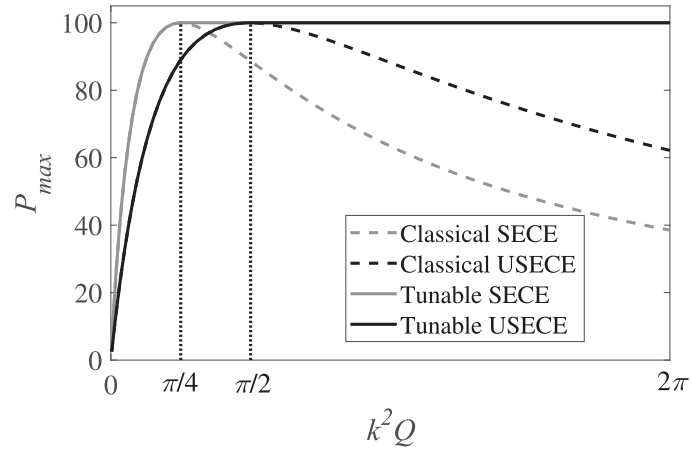

Figure 5. Evolution of the maximum normalized power for classical SECE, classical USECE, tunable SECE and tunable USECE with respect to the FOM $k^{2} Q(Q=100)$.

the tuning parameter $\beta$. As for tunable SECE (Richter et al., 2014), a simple interface can provide an arbitrary (but constant) $\beta$ independent of the oscillation frequency. One may wonder the performances that a tunable USECE would provide if the tuning factor $\beta$ is set at the maximum of $\beta$ (called $\beta_{\max }$ ) with respect to the frequency $\Omega$ instead of specifically tuned for each $\Omega$. This $\beta_{\max }$ depends on the FOM $k^{2} Q$.

The corresponding results are given in Figure 6. The main impact of this design choice is a narrower bandwidth, especially in the case of a very strong coupling. However, it remains a choice worthy of investigation for two reasons. First, in the weak-coupling case, the reduction of the input power compared to the case of variable $\beta$ is relatively small. Second, in the strong-coupling case, much more power is harvested with $\beta=\beta_{\max }$ than for classical USECE (which corresponds to $\beta=0$ ), for a very limited complexity of implementation.

Now that the theoretical investigations have been performed and confirm the interest and limitations of tunable USECE, we present an experimental validation of the model and control scheme.

\section{Experimental validation}

\section{I. Experimental setup}

The generator used for the experimental validation is a $60 \times 37 \times 0.5 \mathrm{~mm}^{3}$ stainless steel cantilever beam with a glued $36 \times 36 \times 0.3 \mathrm{~mm}^{3}$ PZT-5H plate. The setup is similar to the one developed in Brenes et al. (2018) except that, at each frequency, a control loop tunes the duty-cycle of the pulse-width modulation (PWM) control signal to maximize the input power (step-by-step dichotomy). The schematic of the experimental setup is depicted in Figure 7.

The linearity of the resonator is validated through admittance measurements, reported in Figure 8. The electronic load is simulated by adding a resistor $R_{\text {load }}=50 \mathrm{k} \Omega$. The output capacitor $C_{\text {out }}$ is $4.7 \mu \mathrm{F}$. The inductor is $L=100 \mathrm{mH}$. Typical waveforms obtained with the USECE and tunable USECE are reported in Figure 9. A picture of the experimental setup is given in Figure 10. A picture of the electronic circuit used in the experiments can be found in a previous work (Brenes et al., 2018). As explained in our theoretical investigations, for the same acceleration amplitude, the peak 

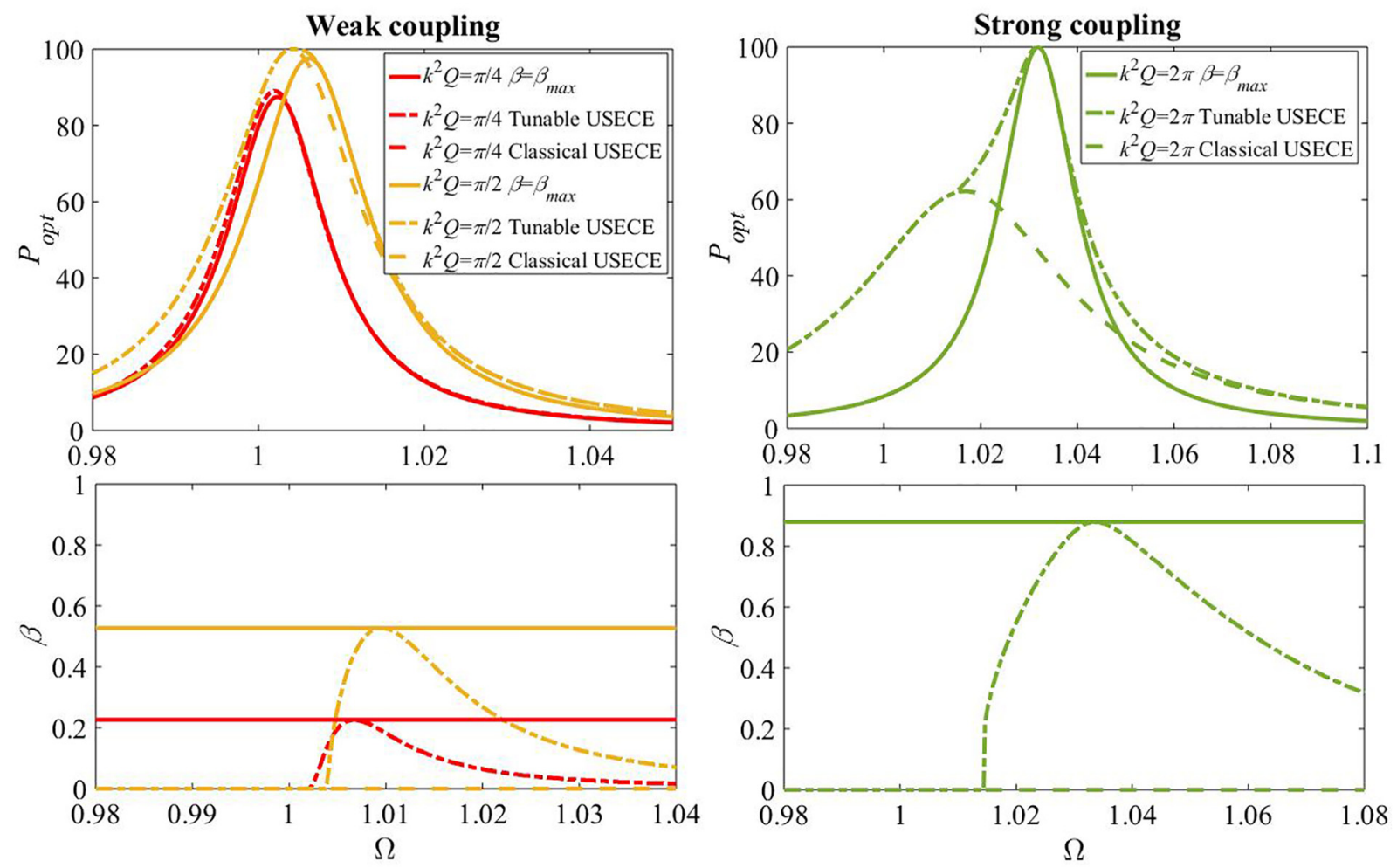

Figure 6. Comparison between classical USECE and tunable USECE with variable $\beta$ and with fixed $\beta=\beta_{\max }$ with $Q=100$. Dashed lines indicate classical USECE, dash-dotted lines indicate tunable USECE and solid lines indicate tunable USECE with $\beta=\beta_{\max }$.

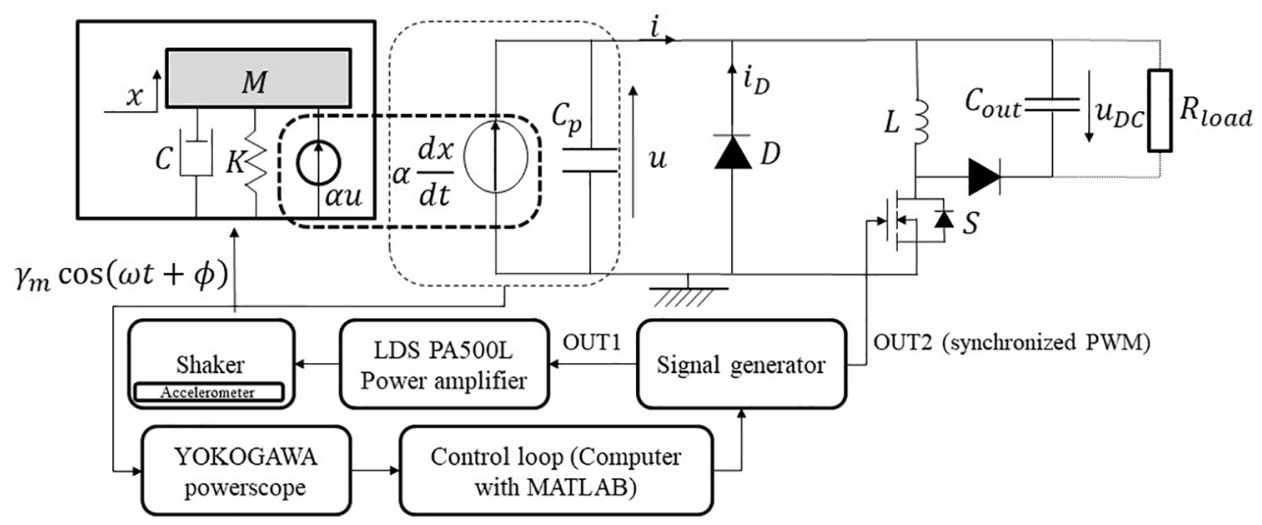

Figure 7. Experimental setup.

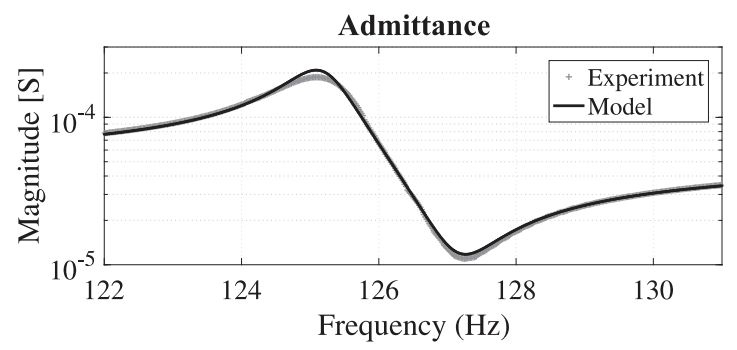

Figure 8. Admittance magnitude: measurements (markers) and fit with a linear model (solid line). voltage of tunable USECE close to resonance is larger than the peak voltage of classical USECE because the amplitude of the motion is larger (Table 2).

\subsection{Model validation}

Measurements of the input power (i.e. the power extracted from the piezoelectric generator) are reported in Figure 11, along with a comparison to theoretical results. The acceleration amplitude is set at $0.2 \mathrm{~g}$ RMS. The FOM of the piezoelectric generator is 


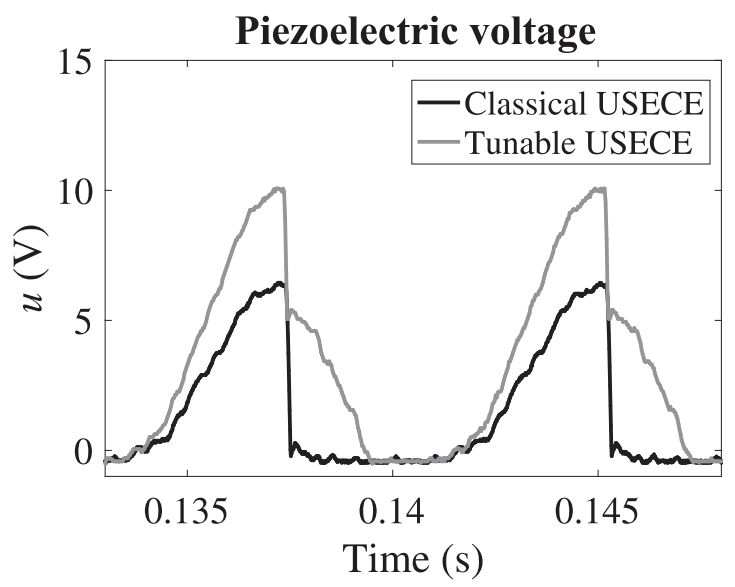

Figure 9. Experimental waveforms of the piezoelectric voltage at $128 \mathrm{~Hz}$ and $0.2 \mathrm{~g}$ RMS acceleration. Grey lines indicate tunable USECE and dark lines indicate classical USECE.

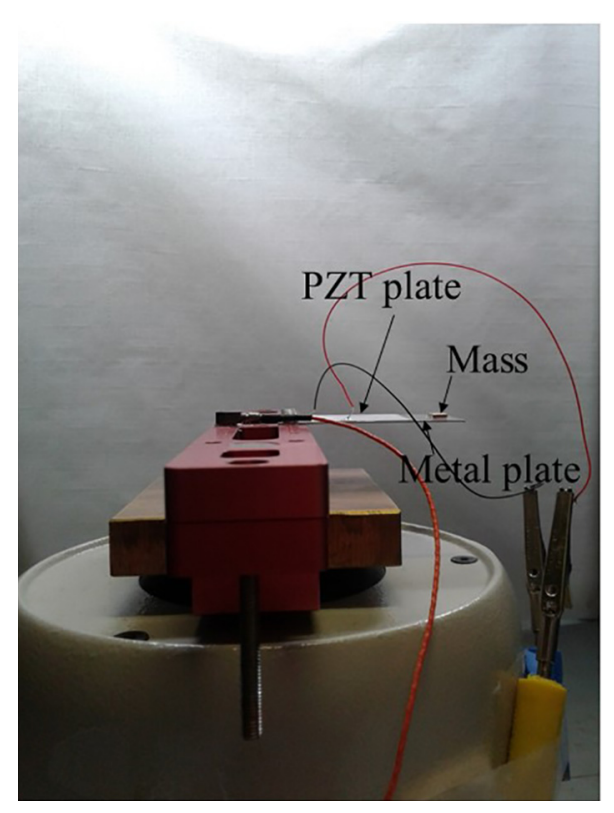

Figure 10. Picture of the experimental setup.

Table 2. Electromechanical parameters.

\begin{tabular}{ll}
\hline Parameter & Estimated value \\
\hline$C_{p}$ & $62 \mathrm{nF}$ \\
$f_{0}$ & $125.2 \mathrm{~Hz}$ \\
$M$ & $5.0 \mathrm{~g}$ \\
$\alpha$ & $2.46 \times 10^{-3} \mathrm{NV}^{-1}$ \\
$k^{2}$ & $3.0 \times 10^{-2}$ \\
$Q$ & 100 \\
\hline
\end{tabular}

$k^{2} Q \simeq 3>\pi / 2$. With such a FOM, tunable USECE reaches the maximum available power whereas classical USECE does not, as can be seen in Figure 11. The maximal power is increased by approximately $+10 \%$
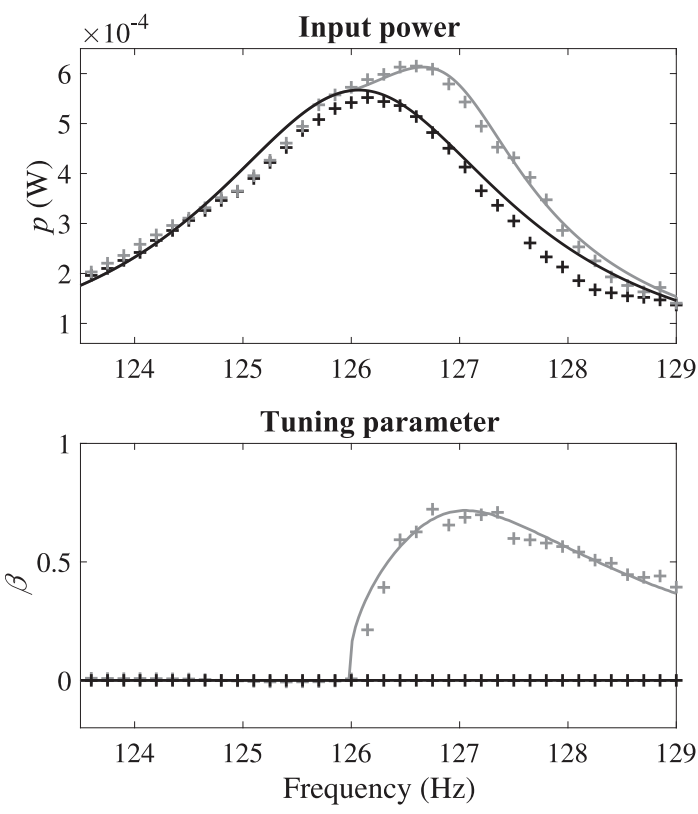

Figure I I. Extracted power of classical USECE (dark) and tunable USECE (grey) at $0.2 \mathrm{~g}$ RMS acceleration with corresponding tuning parameter and output power. Crosses represent experimental results and solid lines indicate theory.

compared to classical USECE and the bandwidth is slightly enlarged. This validates our model and the interest of tunable USECE.

\section{Conclusion}

In this article, we have presented a new control scheme for the recently studied USECE interface used for piezoelectric energy harvesting. A thorough analysis of the piezoelectric coupling shows how tuning the duration of charge extraction increases the maximum harvested power and the bandwidth of the overall system. The resulting architecture, called tunable USECE (due to its similitudes with tunable SECE), yields specific frequency responses able to achieve the maximum available power as soon as $k^{2} Q \geqslant \pi / 2$. A significant gain especially appears for strongly coupled generators, and the gain compared to non-tunable architectures gets larger as the coupling gets stronger.

The performance of the proposed control scheme has been demonstrated experimentally with an automated control loop algorithm based on a step-by-step dichotomy maximizing the power flow from the generator into the electronics. The implementation of the tunable USECE interface with analog and/or digital electronic components in a fully embedded system is the subject of ongoing research.

\section{Declaration of conflicting interests}

The author(s) declared no potential conflicts of interest with respect to the research, authorship, and/or publication of this article. 


\section{Funding}

The author(s) disclosed receipt of the following financial support for the research, authorship, and/or publication of this article: This work was supported by the Korea Institute of Energy Technology Evaluation and Planning (KETEP) and the Ministry of Trade, Industry \& Energy (MOTIE) of the Republic of Korea (No. 20158510060040).

\section{ORCID iD}

Alexis Brenes (iD https://orcid.org/0000-0002-3052-1179

\section{References}

Arroyo E, Badel A, Formosa F, et al. (2012) Comparison of electromagnetic and piezoelectric vibration energy harvesters: model and experiments. Sensors Actuators A: Physical 183: $148-156$.

Badel A and Lefeuvre E (2016) Nonlinear conditioning circuits for piezoelectric energy harvesters. In: Blokhina E, Aroudi AE, Alarcon E and et al. (eds) Nonlinearity in Energy Harvesting Systems. Cham: Springer, pp. 353-357.

Boussaid SLF (2015) A highly efficient P-SSHI rectifier for piezoelectric energy harvesting. IEEE Transactions on Power Electronics 30(10): 5364-5369.

Brenes A, Lefeuvre E, Badel A, et al. (2018) Unipolar synchronized electric charge extraction for piezoelectric energy harvesting. Smart Materials and Structures 27: 075054.

Gasnier P, Willemin J, Boisseau S, et al. (2014) An autonomous piezoelectric energy harvesting IC based on a synchronous multi-shots technique. IEEE Journal of SolidState Circuits 49(7): 1561-1570.

Gelb A and Velde WEV (1968) Multiple-input Describing Functions and Nonlinear System Design. Cambridge: McGraw-Hill Education.

Guyomar D and Lallart M (2011) Recent progress in piezoelectric conversion and energy harvesting using nonlinear electronic interfaces and issues in small scale implementation. Micromachines 2: 274-294.

Halvorsen E, Le CP, Mitcheson PD, et al. (2013) Architecture-independent Power Bound for Vibration Energy Harvesters. London: Journal of Physics, Conference Series.

Kazimierczuk MK (1990) Analysis of class E zero-voltageswitching rectifier. IEEE Transactions on Circuits and Systems 37(6): 747-755.

Lallart M (2017) Nonlinear technique and self-powered circuit for efficient piezoelectric energy harvesting under unloaded cases. Energy Conversion and Management 133: 444-457.
Lefeuvre E, Badel A, Brenes A, et al. (2017a) Analysis of piezoelectric energy harvesting system with tunable SECE interface. Smart Materials and Structures 26(3): 035065.

Lefeuvre E, Badel A, Brenes A, et al. (2017b) Power and frequency bandwidth improvement of piezoelectric energy harvesting devices using phase-shifted SECE interface circuit. Journal of Intelligent Material Systems and Structures 28(20): 2988-2995.

Lefeuvre E, Badel A, Richard C, et al. (2005) Piezoelectric energy harvesting device optimization by synchronous electric charge extraction. Journal of Intelligent Material Systems and Structures 16: 865-876.

Lesieutre GA, Ottman GK and Hofmann HF (2004) Damping as a result of piezoelectric energy harvesting. Journal of Sound and Vibration 269: 991-1001.

Li K (2011) Structural Vibration Damping with Synchronized Energy Transfer between Piezoelectric Patches. Lyon: Lyon INSA.

Liao Y and Sodano HA (2008) Model of a single mode energy harvester and properties for optimal power generation. Smart Materials and Structures 17: 065026.

Lien IC, Shu YC, Wu W-J, et al. (2010) Revisit of series-SSHI with comparisons to other interfacing circuits in piezoelectric energy harvesting. Smart Materials and Structures 19: 125009.

Richter A, Strobel A, Joram N, et al. (2014) Tunable interface for piezoelectric energy harvesting. In: IEEE 11th International Multi-Conference on System, Signals \& Devices (SSD14), Barcelona, 11-14 February, New York: IEEE.

Sanchez DA, Leicht J, Jodka E, et al. (2016) A $4 \mu w-t o-1 m w$ parallel-SSHI rectifier for piezoelectric energy harvesting of periodic and shock excitations with inductor sharing, cold start-up and up to $681 \%$ power extraction improvement. In: IEEE International Solid-State Circuits Conference (ISSCC), San Francisco, CA, 31 January-4 February, New York: IEEE.

Shu YC and Lien IC (2006) Analysis of power output for piezoelectric energy harvesting systems. Smart Materials and Structures 15: 1499-1512.

Wu Y, Badel A, Formosa F, et al. (2014) Self-powered optimized synchronous electric charge extraction circuit for piezoelectric energy harvesting. Journal of Intelligent Material Systems and Structures 25: 2165-2176.

Yoshimizu K, Yamamoto Y, Asahina K, et al. (2017) Strategy for enhancing the active harvesting of piezoelectric energy. Journal of Intelligent Material Systems and Structures 28(8): 1059-1070. 